\title{
The effect of cognitive training on the subjective perception of well-being in older adults
}

\author{
Vladimír Bureš $^{1}$, Pavel Čech ${ }^{\text {Corresp., }}{ }^{1}$, Jaroslava Mikulecká ${ }^{1}$, Daniela Ponce ${ }^{1}{ }^{\text {, }}$ Kamil Kuca ${ }^{1,2}$ \\ 1 Faculty of Informatics and Management, University of Hradec Kralove, Hradec Kralove, Czech Republic \\ 2 Biomedical Research Centre, University Hospital Hradec Kralove, Hradec Kralove, Czech Republic \\ Corresponding Author: Pavel Čech \\ Email address: pavel.cech@uhk.cz
}

Background. There is a growing number of studies indicating the major consequences of the subjective perception of well-being on mental health and healthcare use. However, most of the cognitive training research focuses more on the preservation of cognitive function than on the implications of the state of well-being. This secondary analysis of data from a randomised controlled trial investigated the effects of individualised television-based cognitive training on self-rated well-being using the WHO-5 index while considering gender and education as influencing factors. The effects of cognitive training were compared with leisure activities that the elderly could be engaged in to pass time.

Methods. Cognitively healthy participants aged 60 years or above screened using the Mini-Mental State Examination (MMSE) and Major Depression Inventory (MDI) were randomly allocated to a cognitive training group or to an active control group in a single-blind controlled two-group design and underwent 24 training sessions. Data acquired from the WHO-5 questionnaire administered before and after intervention were statistically analysed using a mixed design model for repeated measures. The effect of individualised cognitive training was compared with leisure activities while the impact of gender and education was explored using estimated marginal means.

Results. A total of 81 participants aged $67.9 \pm 5.59$ [60-84] without cognitive impairments and absent of depression symptoms underwent the study. Participants with leisure time activities declared significantly higher scores compared to participants with cognitive training $\mathrm{M}=73.48 \pm 2.88,95 \% \mathrm{Cl}$ $[67.74,79.22]$ vs $M=64.13 \pm 3.03,95 \% \mathrm{Cl}[58.09,70.17]$. Gender and education were found to moderate the effect of cognitive training on well-being when compared to leisure activities. Females engaged in leisure activities in the control group reported higher by $\mathrm{M}=9.77 \pm 5.4,95 \% \mathrm{Cl}[-0.99$, 20.54] WHO-5 scores than females with the cognitive training regimen. Participants with high school education declared leisure activities to increase WHO-5 scores by $\mathrm{M}=14.59 \pm 5.39,95 \% \mathrm{Cl}[3.85$, 25.34] compared to individualised cognitive training.

Discussion. The findings revealed that individualised cognitive training was not directly associated with improvements in well-being. Changes in the control group indicated that involvement in leisure time activities, in which participants were partly free to choose from, represented more favourable stimulation to a self-perceived sense of well-being than individualised cognitive training. Results also supported the fact that gender and education moderated the effect of cognitive training on well-being. Females and participants with high school education were found to be negatively impacted in well-being when performance connected with cognitive training was expected. 
1 The Effect of Cognitive Training on the Subjective Perception of Well2 Being in Older Adults

3 Vladimir Bures, ${ }^{1}$ Pavel Cech, ${ }^{1}$ Jaroslava Mikulecka, ${ }^{1}$ Daniela Ponce, ${ }^{1}$ Kamil Kuca ${ }^{1,2}$

$4 \quad{ }^{1}$ Faculty of Informatics and Management, University of Hradec Kralove, Hradec Kralove, Czech

5 Republic

$6 \quad{ }^{2}$ Biomedical Research Centre, University Hospital Hradec Kralove, Hradec Kralove, Czech

7 Republic

8

9 Corresponding author:

10 Pavel Čech,

11 Rokitanského 62, Hradec Kralové 500 03, Czech Republic

12 Email: pavel.cech@uhk.cz 


\section{Abstract}

14 Background. There is a growing number of studies indicating the major consequences of the subjective perception of well-being on mental health and healthcare use. However, most of the cognitive training research focuses more on the preservation of cognitive function than on the implications of the state of well-being. This secondary analysis of data from a randomised controlled trial investigated the effects of individualised television-based cognitive training on self-rated well-being using the WHO-5 index while considering gender and education as influencing factors. The effects of cognitive training were compared with leisure activities that the elderly could be engaged in to pass time.

Methods. Cognitively healthy participants aged 60 years or above screened using the MiniMental State Examination (MMSE) and Major Depression Inventory (MDI) were randomly allocated to a cognitive training group or to an active control group in a single-blind controlled two-group design and underwent 24 training sessions. Data acquired from the WHO-5 questionnaire administered before and after intervention were statistically analysed using a mixed design model for repeated measures. The effect of individualised cognitive training was compared with leisure activities while the impact of gender and education was explored using estimated marginal means.

Results. A total of 81 participants aged $67.9 \pm 5.59$ [60-84] without cognitive impairments and absent of depression symptoms underwent the study. Participants with leisure time activities declared significantly higher scores compared to participants with cognitive training $\mathrm{M}=73.48 \pm$ $2.88,95 \%$ CI $[67.74,79.22]$ vs $\mathrm{M}=64.13 \pm 3.03,95 \%$ CI [58.09, 70.17]. Gender and education were found to moderate the effect of cognitive training on well-being when compared to leisure activities. Females engaged in leisure activities in the control group reported higher by $\mathrm{M}=9.77$ $\pm 5.4,95 \%$ CI $[-0.99,20.54]$ WHO-5 scores than females with the cognitive training regimen.

37 Participants with high school education declared leisure activities to increase WHO-5 scores by $\mathrm{M}=14.59 \pm 5.39,95 \% \mathrm{CI}[3.85,25.34]$ compared to individualised cognitive training.

Discussion. The findings revealed that individualised cognitive training was not directly associated with improvements in well-being. Changes in the control group indicated that involvement in leisure time activities, in which participants were partly free to choose from, 
42 represented more favourable stimulation to a self-perceived sense of well-being than

43 individualised cognitive training. Results also supported the fact that gender and education

44 moderated the effect of cognitive training on well-being. Females and participants with high

45 school education were found to be negatively impacted in well-being when performance

46 connected with cognitive training was expected.

\section{Introduction}

48 There is evidence that cognitive training has a positive effect on cognitive function, and the

49 preservation of cognitive function is associated with a healthier lifestyle and greater well-being

50 (Henry et al., 2015; Llewellyn et al., 2008; WHO, 2011). However, recent studies on computer-

51 based cognitive training have focused mainly on improvements in cognitive function or transfer

52 to other cognitive tasks (Kueider et al., 2012; Maseda et al., 2013; Rizkalla, 2015; Shatil et al.,

53 2014; van Muijden et al., 2012) rather than on the self-perceived emotional experience denoted

54 as well-being. Therefore, the effect of cognitive training on psychological well-being remains

55 unclear. Cognitive training is believed to positively influence well-being as a consequence of

56 preserving the cognitive function (Adamski et al., 2016; Innes et al., 2016; Smith, 2016) but this

57 relationship is assumed rather than supported by evidence. Correspondingly, not much is known

58 about factors that might influence the observed effect of cognitive training on well-being.

59 The rationale for drawing attention to well-being is due to studies indicating the major

60 consequences of well-being and of positive mental health for a healthier life, improved

61 healthcare use and corresponding social outcomes (Allerhand et al., 2014; Buiza et al., 2009;

62 Walker \& Lowenstein, 2009). In health, well-being denotes optimal psychological functioning

63 and experience (Ryan \& Deci, 2001) and consists of the ability to be happy or at least contended

64 and have the capacity that enhances self-esteem, maintains beliefs in personal efficacy, and

65 promotes an optimistic view of the future (Taylor \& Brown, 1988). Well-being is argued to

66 complement functional and health status in assessing patients' quality of life and is considered as

67 a proxy indicator of quality of life in response to a treatment, intervention and also to ageing

68 (Brown et al., 2004; Eiser \& Morse, 2001).

69 Even normally aging older adults experience significant impairment in attentional tasks

70 (Getzmann et al., 2016; Sperduti et al., 2016), episodic memory (Manenti et al., 2016; Tromp et 
71 al., 2015) and working memory tasks (Jost et al., 2011; Ko et al., 2014), time perception

72 (Turgeon et al., 2016) and also speed of processing (Ball et al., 2013; Elgamal et al., 2011). If

73

74

75

76

77

poorly prevented, these impairments might degrade the quality of life as well as the psychological well-being (Pusswald et al., 2015; Stogmann et al., 2015). Other studies have also indicated that cognitive decline in its early stages often goes unnoticed by individuals as the activities of daily living in familiar environments are not affected (Calzà et al., 2015; Missotten et al., 2008; Ready et al., 2003) and these studies further stress the importance of prevention in order to maintain cognitive abilities and well-being.

There is evidence that women feel worse than men in terms of subjective well-being (Stevenson \& Wolfers, 2009), yet women are reported to declare higher levels of well-being when they experience similar conditions to men (Senik, 2015). The subjective well-being gap between women and men in high-income countries is explained by women having different uses of their time, lower expectations especially concerning their social role and richer social ties (Graham \& Chattopadhyay, 2013; Lun \& Bond, 2016). The gender gap in well-being could also be related to differences in mental health in men and women. According to the World Health Organization, gender is a critical determinant of mental health and mental illness (WHO, 2015). Unipolar depression, for instance, is twice as common in women and gender has also been found to be a significant predictor of psychotropic drug prescription (Nana et al., 2015; Simoni-Wastila, 2000).

Rarely is the impact of education on well-being in connection with cognitive training in elderly population addressed (as evidenced by search in PubMed databases dated to October 2016).

Nevertheless, studies on cognitive training suggest that specific cognitive exercises are moderated by educational attainment. For instance, the study of Willis and Caskie (2013) reports that higher education is related to higher baseline scores in cognitive training exercises. However, participants with lower levels of education exhibited greater training effects in Letter and Word Series exercises (Willis \& Caskie, 2013). Similarly, a recent study by Clark at al. (2015) shows that the effect of cognitive training on cognitive function does not differ in memory and reasoning exercises by educational attainment but it is significantly different in the speed of processing. The effect of speed of processing training is $50 \%$ greater in normal older adults with a less than complete high school education (Clark et al., 2015). In contrast, Maseda et al. (2013) point out that the effect of cognitive training was less pronounced for subjects with 
101 incomplete primary studies. Given the assumed link between improved cognitive function and

102 improved well-being, the conjecture in the present study is that the education might moderate

103 also the effect of cognitive training on well-being.

104 Recently, there were attempts to study well-being in relation to meditation (Goyal et al., 2014),

105 yoga involvement (Gaiswinkler \& Unterrainer, 2016), physical exercise such as running (Skead

$106 \&$ Rogers, 2016) or whole body movements (Ben-Soussan et al., 2015), social engagement

107 (Rapacciuolo et al., 2016) or intergeneration social support (Tian, 2016). These studies prove

108 that well-being might be stimulated by consciously focusing on the positive side of life, by

109 fostering an active and healthy life-style and by encouraging social ties. In respect to cognitive

110 function Allerhand et al. (2014) pointed out that higher levels of well-being are associated with

111 better cognitive performance and also that above average cognitive function implies greater well-

112 being. The positive bidirectional relationship of cognitive function and well-being supports the

113 assumed effect of cognitive training. However, cognitive training studies do not prove this link.

114 There are studies on cognitive training in which positive effect on well-being is often implicitly

115 inferred based on improvements in cognitive function but not explicitly measured (Adamski et

116 al., 2016; Gates et al., 2011; Innes et al., 2016; Smith, 2016). In a study by Jansen and Dahmen-

117 Zimmer (2012) the effect of cognitive training on well-being is analysed in combination with

118 motor and karate training. However, they used the Anxiety-Depression Scale instead of scales

119 designed to measure well-being. It has already been pointed out that, although depression is

120 associated with lower levels of well-being, there is increasing evidence that positive well-being

121 and negative mental states are to some degree independent (Allerhand et al., 2014; Huppert \&

122 Whittington, 2003; Isaacowitz \& Smith, 2003). There are also cognitive training studies that

123 focus on people with already developing mental problems such as mild cognitive impairments

124 (Belleville et al., 2006; Bier et al., 2015) or memory complaints (Cohen-Mansfield et al., 2015).

125 A majority of the studies does not clearly explicate the effect of cognitive training on well-being

126 in a population of healthy elderly adults. Therefore, the present research elaborates on the

127 following research question: What will be the difference in the self-perceived sense of well-

128 being of elderly people involved in individualised cognitive training in regard to gender or

129 education? Studying the effect of cognitive training on the subjective perception of well-being

130 might help in understanding how older people perceive the cognitive training as a part of their

131 everyday lives and what the implications for training adherence are. The aim of this study is to 
132 investigate if there is a statically significant effect of individualised television-based cognitive

133 training on self-perceived well-being based on the WHO-5 index while controlling for education

134 or gender. The effect of cognitive training is compared with a control group engaged in leisure

135 time activities that elderly adults can do to pass the time.

137 Materials and Methods

\section{Study design}

139 The study design was based on secondary analysis of a randomised controlled trial conducted at

140 the facilities of the University of Hradec Kralove, in the East Bohemia Region, Czech Republic.

141 The trial was part of the project Vital Mind (European Union 7th Framework Programme,

142 project number ICT-215387) that focused on migrating computer-based cognitive training to a

143 digital television platform. The European Commission granted ethical approval to carry out the

144 study within the scope of the Vital Mind project by accepting the Ethical Road Map report

145 (deliverable D8.6.1). The institutional ethical approval was granted by the chancellor's office of

146 the University of Hradec Kralove (Vital Mind ref. no. 215387). The study was undertaken with

147 the understanding and written informed consent of each participant and according to the World

148 Medical Association Declaration of Helsinki ethical principles. The study was designed to

149 consist of 24 sessions over the course of eight weeks, i.e. three sessions per week were

150 administered to the participants. Each session was supposed to last 20 to 30 minutes, totalling 8

15112 hours. The sessions were held during the morning and afternoon (from 8 a.m. till 4 p.m.) in

152 special rooms. There were four rooms operating in parallel in order to serve all the participants.

153 Each session was assisted by trained personnel. The aim was to provide administrative and

154 technical assistance. During the training part of the session, the assistant interfered only in

155 situations in which the participants were in doubt about how to continue or were having

156 problems with the technology.

\section{Participants}

158 The sample consisted of 140 older adults aged 60+ from the East Bohemia Region. The

159 candidates were recruited via advertisements in all local newspapers and by a recruitment

160 procedure at the University of Lifelong Learning. The University of Lifelong Learning is a 
161 public institute affiliated with the University of Hradec Kralove that provides further education 162 to more than 1,500 attendees every year. During the recruitment procedure at the University of

163 Lifelong Learning, information about the study was announced during regular courses and the 164 contact details of prospective volunteers were collected. Volunteers were then invited for a brief 165 introduction to the study after which they were asked to confirm their interest in participating in 166 the study.

167 This study focused only on retired people who do not live in community centres or care homes.

168 The eligibility assessment was conducted under the supervision of a psychologist using the Mini169 Mental State Examination (MMSE) (Folstein et al., 1975) to test for cognitive impairment and 170 Major Depression Inventory (MDI) (Bech et al., 2001; Olsen et al., 2003) to screen for 171 depressive symptoms. The inclusion criteria were the following: age $\geq 60$, MMSE $\geq$ median 172 values adjusted for age and education reported by (Crum et al., 1993), absence of moderate to 173 severe depressive symptoms based on ICD-10 algorithm (Bech et al., 2001), no assistance 174 required in daily routines, no motor, visual and hearing impairments that would hinder the 175 training. Based on the inclusion criteria, 81 participants were admitted to take part in the study.

176 The participants were informed that finishing all planned sessions would be financially rewarded 177 with $€ 100$ approx. to cover travel and other 'out-of-pocket' expenses.

178 Participants were randomly divided into two groups: an active control group (referred to as 179 control group hereafter) and a cognitive group with leisure time activities and with individualised 180 cognitive training respectively. The randomisation procedure was carried out using computer generated pseudorandom numbers assigned to each participant. The generated numbers were

182 then randomly divided into the two given groups. To avoid placebo like effects and motivation 183 distortion described by Boot, Blakely and Simons (2011) in which people in a cognitive group 184 perform better because they are expected to do so, participants were not aware of the distinction 185 between the groups and the aim of the research was generalised to both groups (single-blind 186 controlled two-group design).

\section{Materials}

188 The MMSE (Folstein et al., 1975) in the Czech version (Brazdil et al., 1995) was used as a 189 screening tool for cognitive impairment and the MDI (Bech et al., 2001; Olsen et al., 2003) was 190 used to indicate depressive symptoms. Both the MMSE and MDI tests were administered by 
191 trained personnel (senior researchers) under the supervision of a psychologist. The MMSE cut-

192 off score of 24 was suggested by Folstein et al. (1975), however, later studies showed that the

193 MMSE score was moderated by age and education (Crum et al., 1993; O'Bryant et al., 2008).

194 There have been no validation study with normative scores of MMSE stratified by age and years

195 of education in Czech population published in a widely prestigious international journal,

196 therefore, the present study adopted median values reported by Crum et al. (1993) as cut-off

197 scores. The MDI answers were dichotomised based on the ICD-10 algorithm in which moderate

198 to severe depression is observed if at least two out of three core symptoms are present as well as

199 at least four of the other seven items (Bech et al., 2001). The WHO-5 Well-Being Index was

200 used for well-being screening. The WHO-5 Well-Being Index covering five positively worded

201 items, related to positive mood, vitality and general interests, has been shown to be a reliable

202 measure of emotional functioning and a good screener of depression. The raw score ranges are

203 from 0 to 25, with higher scores indicating better well-being. The raw score is transformed to 0

204100 scale by multiplying by 4 . In order to measure possible changes in well-being, a $10 \%$

205 difference can be regarded as a practically significant change (Topp et al., 2015). The Czech

206 version (WHO, 1998) was used in the present study. The WHO-5 was selected because it is short

207 and valid for the participants' age group (McDowell, 2010), robust (Bech, 2012), suitable for a

208 mentally healthy sample, and measures positive affect (Taylor \& Brown, 1988).

209 Intervention

210 The cognitive group was involved in special cognitive tasks such as reaction speed, the time to

211 respond to a simple stimulus (when no decision was involved), the ability to remember

212 information for a short period of time, the ability to split attention between two or more channels

213 of information, the ability to estimate event duration, the ability to retrieve a word from the

214 semantic lexicon, awareness of the channel (source) of knowledge or information, planning as

215 the ability to mentally anticipate the correct way (think ahead) to execute a task, or the ability to

216 grasp quickly the meaning of a visual stimulus. During the first session participants were tested

217 using 11 cognitive tasks. The results of those tasks were used to individualise training programs

218 based on the specific needs of each participant in the cognitive group. Each session of the

219 training program was further adjusted according to the progress of a given participant so that the

220 perceived intensity of the training program is equalized. The training program was validated in

221 (Gigler et al., 2013; Peretz et al., 2011; Shatil, 2013). The training program was embedded in 
222 digital television and allowed participants to interact with the training program in a familiar

223 manner. Television as a low-cost training device was assessed with favourable results in Boquete

224 et al. (2011). Neither touch screens nor sensory controls were used. Participants followed the

225 training program fairly strictly.

226 The active control group was engaged in leisure activities as if passing time at home. The control

227 group activities were not restricted to non-cognitive tasks and might be cognitively challenging

228 but were strictly different from the cognitive group exercises in that: a) they were not

229 individualised; b) they did not follow any specific cognitive training protocol. The control group

230 activities included building a family tree (they had the opportunity to bring their own photos,

231 digitalise them and build their own family tree), image processing and painting, simple gaming

232 using a pentomino application and also Nintendo sport games. Television guided physical

233 exercise was also included in the schedule. During some sessions, participants could select

234 whatever kind of activity appealed to them.

235 Sessions in both groups were assisted by trained personnel to provide for the proper execution of

236 the intervention. The task of the assistants was to introduce and conclude the session. The

237 assistants also provided technical support if necessary. All assistants were instructed to interfere

238 in the intervention activities only in situations when participants were not able to continue on

239 their own. The digital television-based training application retained the progress scores of the

240 participants in the cognitive group. All participants kept diaries and wrote comments about how

241 much they liked each intervention session and also about their mood between sessions. They also

242 documented mental activities outside the training protocol that might influence their mood.

243 Usually, at the end of each session, assistants questioned all participants in both groups about

244 their mood status and the mood status was recorded in diaries.

245 If participants were not able to attend a planned session they could complete it on the same day

246 at a different time. In some special cases, an extra session was added at the end of the

247 intervention program. In the control group, there were no strict programs to follow. In the

248 cognitive group, participants were instructed to adhere to the individualised training program and

249 there was an insistence on adherence. 


\section{Statistical analysis}

251 The IBM SPSS and R statistical packages were used to conduct the statistical analyses.

252 Descriptive statistics were computed to check if there were any significant differences (at the 253 alpha level 0.05) in demographic characteristics between the cognitive group and the control

254 group. The continuous baseline demographic characteristics were analysed using the independent 255 samples t-test. The nominal and the ordinal demographic characteristics were tested using the 256 Chi Square statistic.

257 A mixed effect model for repeated measures was used to evaluate differences in the WHO-5 258 scores within and between the groups. The dependent variable was the WHO-5 score; the 259 independent variables were Time (pre-test and post-test) as within subject factor and Group 260 (Control or Cognitive), Gender (Male or Female), Education level (High school or University) as 261 between subject factors. The model allowed for the assessment of interventions in post-test 262 scores between the two groups, differences between baseline and post-test scores within each 263 group, and whether there was a significant interaction between the independent variables 264 (whether the impact of one variable was influenced by the level of a second variable). The model 265 also reported the main effect of one independent variable while controlling for the effect of other 266 independent variables. The outcomes were further analysed using marginal means and pairwise 267 comparisons with Least Significance Difference (LSD) test. The mean differences of the within 268 subject variable (Time) were also studied. Differences with p-values $<0.05$ were considered 269 statistically significant. In order to measure real changes in WHO-5 score, a $10 \%$ difference (out 270 of 100) can be regarded as a practically significant change (WHO 1998).

271 The effect size (also known as strength of association) was measured by partial eta squared 272 statistics indicating the proportion of variance explained by the independent variable. The 273 guidelines proposed by Cohen et al. (2002) for interpreting this value are: $0.01=$ small effect, $2740.06=$ medium effect, $0.14=$ large effect.

\section{Results}

\section{Participants in the final analysis}

277 The final statistical analysis included participants who passed the eligibility criteria and finished

278 the whole intervention (i.e. completed all the sessions). The interventions were completed by 117 
279 participants (60 in the cognitive group, 57 in the control group). Due to personal reasons

280 (especially frequent scheduling of sessions and lack of interest) 9 participants (7\%) withdrew

281 from the study (3 from the cognitive group (5\%) and 6 from the control group (9\%). The study

282 eligibility criteria were met by 81 participants (37 in the cognitive group, 44 in the control group)

283 aged $67.9 \pm 5.59$ [60-84]. None of the participants experienced major technical difficulties with

284 using the television as a training device. Table 1 shows that there were no significant differences

285 in baseline personal characteristics in the two groups.

\section{Table 1: Baseline characteristics of participants}

\begin{tabular}{llll}
\cline { 2 - 4 } & $\begin{array}{l}\text { Cognitive Training } \\
\text { Group } \\
(N=37)\end{array}$ & $\begin{array}{l}\text { Control } \\
\text { Group } \\
(N=44)\end{array}$ & $p$ value \\
\hline Age in years (mean $\pm \mathrm{SD}$ ) [range] & $67.8 \pm 5.48[60-79]$ & $68.0 \pm 5.74[61-84]$ & 0.880 \\
Female (\%) & 62.2 & 59.1 & 0.778 \\
University level education (\%) & 48.6 & 34.1 & 0.184 \\
Formal education in years (mean $\pm \mathrm{SD})$ & $15.9 \pm 2.87[11-23]$ & $14.8 \pm 3.14[11-23]$ & 0.108 \\
[range] & $29.6 \pm 0.64[28-30]$ & $29.4 \pm 0.73[28-30]$ & 0.2153 \\
MMSE (mean $\pm \mathrm{SD})$ [range] & $66.7 \pm 18.50[24-100]$ & $65.7 \pm 18.37[8-96]$ & 0.8132 \\
WHO-5 score (mean $\pm \mathrm{SD})$ [range] & & &
\end{tabular}

None of the statistics were significant at the $p<0.05$ level

288

289

290

291

292

293

294

295

296

297

298

299

300

Legend: The table shows baseline characteristics of participants. None of the statistics were significant at the $\mathrm{p}<0.05$ level i.e. participants in both groups were well matched according to age, gender, education, MMSE, and WHO-5 baseline scores.

\section{Effects of individualised cognitive training and leisure activities on well-being}

There was significant interaction between Group and Time, Wilks' Lambda $=0.992, \mathrm{~F}(1,73)=$ $0.563, \mathrm{p}=0.018$, partial eta squared $=0.074$ (small effect). The interaction indicated that there were significant differences in how participants responded in terms of WHO-5 scores to interventions across the groups regardless of gender and education level. The estimated marginal means revealed that participants in the cognitive group reported lower WHO-5 scores (Pre-test $\mathrm{M}=67.21 \pm 3.19,95 \% \mathrm{CI}[60.86,73.56]$, Post-test $\mathrm{M}=64.13 \pm 3.03,95 \% \mathrm{CI}[58.09,70.17])$ while participants in the control group reported higher WHO-5 scores (Pre-test $\mathrm{M}=66.8 \pm 3.03$, $95 \%$ CI [60.77, 72.83], Post-test $\mathrm{M}=73.48 \pm 2.88,95 \%$ CI [67.74, 79.22]) (Fig. 1). Pairwise comparison of the means using the LSD procedure revealed that the increased WHO-5 scores 
301 (Pre-test to Post-test) in the control group were statistically significant (minimum mean

302 difference $\mathrm{M}=6.68 \pm 2.78,95 \% \mathrm{CI}[1.14,12.22], \mathrm{p}=0.019)$. The reduced WHO-5 scores in the

303 cognitive group were not statistically significant. After the intervention, the control group

304 participants declared significantly higher WHO-5 scores (Post-test) than participants in the

305 cognitive group (minimum mean difference $\mathrm{M}=8.55 \pm 3.97,95 \% \mathrm{CI}[0.64,16.46], \mathrm{p}=0.035$ ).

306 Fig. 1: Estimated marginal means for Group*Time

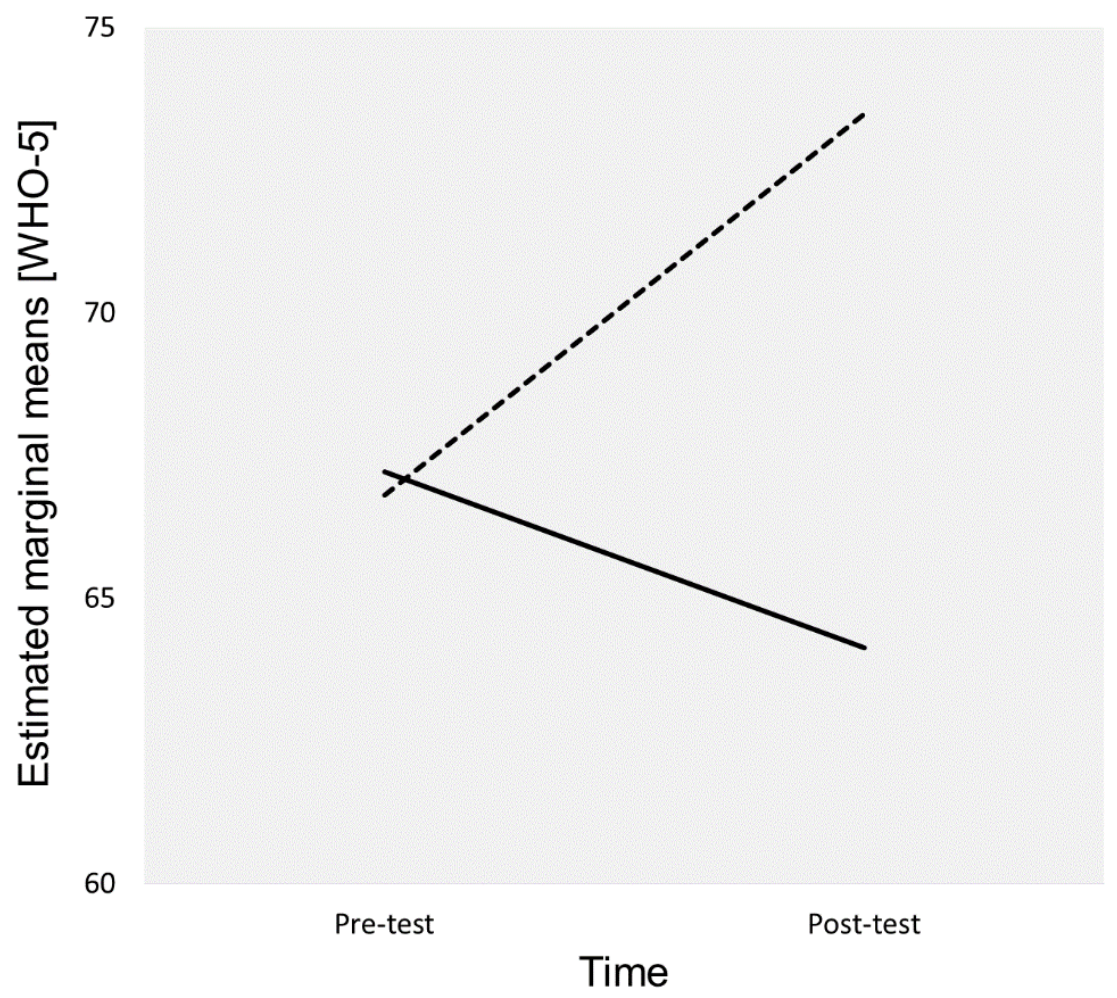

Legend: The plot shows the estimated marginal means (WHO-5) for Time*Group.

310 The mean differences of WHO-5 scores over 8 weeks (Post-test - Pre-test) $\mathrm{M}=6.68 \pm 2.78,95$

$311 \% \mathrm{CI}[1.14,12.22]$ and $\mathrm{M}=-3.08 \pm 2.92,95 \% \mathrm{CI}[-8.9,2.75]$ in control group and cognitive

312 group participants, respectively, were below 10\% threshold of practical significance (Fig. 2).

313 Therefore, there was no practical difference in the WHO-5 scores in any of the groups. 
314 Fig. 2: Mean differences in WHO-5 scores according Group

315

316

317

318

319

320

321

322

323

324

325

326

327

328

329

330

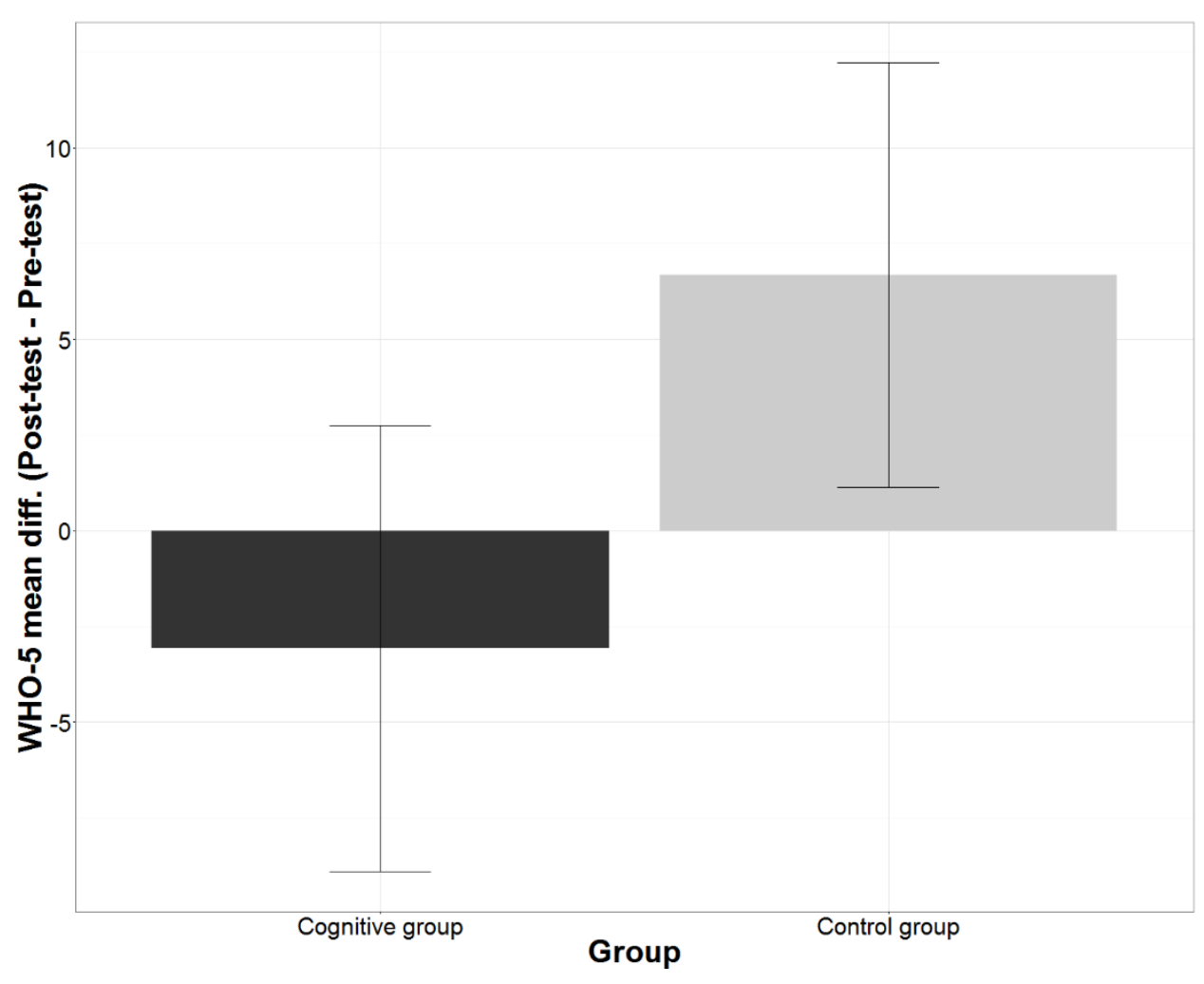

Legend: The figure shows within subject mean differences in WHO-5 scores with 95\% confidence intervals split according the group.

\section{Moderating effect of education}

There was statistically significant interaction between Time and Group among the participants with only high school education, Wilks' Lambda $=0.876, \mathrm{~F}(1,46)=6.538, \mathrm{p}=0.014$, partial eta squared $=0.124$ (medium effect). There was no significant interaction between Time and Group among the university graduates. This indicated that high school education participants were the most effected by the different regimen in the control and the cognitive group $(\mathrm{M}=74.53 \pm 3.47$, $95 \%$ CI $[67.61,81.44]$ vs $\mathrm{M}=59.93 \pm 4.13,95 \% \mathrm{CI}[51.71,68.16])$. The marginal means plot in Fig. 3 illustrates the interaction between time and group among university graduates (Fig. 3A) and high school graduates (Fig. 3B). The plot shows that the university graduates declared increased WHO-5 scores in both groups but in the control group the increase was more pronounced. There was an inverse trend among high school graduates with an increase in WHO-5 scores in the control group but a decrease in the cognitive group. The increase in WHO- 
3315 scores among high school graduates in the control group is statistically significant, Wilks'

332 Lambda $=0.945, \mathrm{~F}(1,73)=4.241, \mathrm{p}=0.043$, partial eta squared $=0.055$ ( small effect).

333 Fig. 3: Estimated marginal means for Time*Education level*Group

A

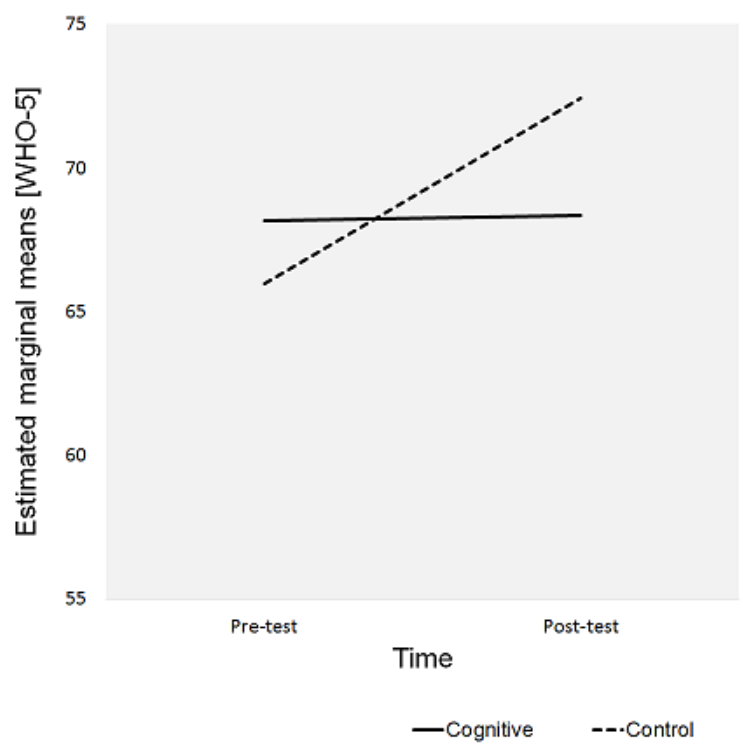

B

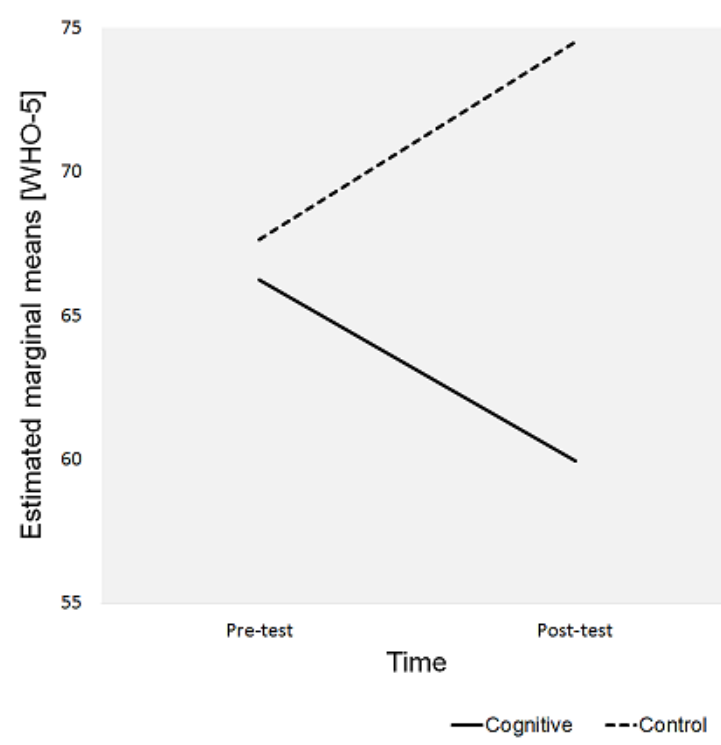

335 Legend: The plots show the estimated marginal means (WHO-5) for Time*Education level*Group. A) Education level $=$ University. B) Education level = High school.

The mean differences of WHO-5 scores over 8 weeks (Post-test - Pre-test) in participants with high school education in the control group were $\mathrm{M}=6.9 \pm 3.35,95 \% \mathrm{CI}[0.22,13.57]$ and WHO-5 scores observed in high school participants in the cognitive group were $\mathrm{M}=-6.32 \pm$ $3.98,95 \%$ CI $[-14.25,1.62]$. The mean differences in WHO-5 scores among university graduates were in the control group $\mathrm{M}=6.46 \pm 4.44,95 \% \mathrm{CI}[-2.38,15.3]$ and in the cognitive 343 group $\mathrm{M}=0.17 \pm 4.28,95 \%$ CI [-8.37, 8.71] (Fig. 4). Pairwise comparison of the means using 344 the LSD procedure revealed that after the intervention (Post-test) high school participants in the control group declared significantly higher WHO-5 scores than high school participants in the cognitive group (minimum mean difference $\mathrm{M}=14.594 \pm 5.392,95 \% \mathrm{CI}[3.85,25.34], \mathrm{p}=$ 0.008). The contrast in mean differences of 14.594 reached the $10 \%$ threshold of practical 
348 significance in WHO-5 scores. The mean differences among university graduates were not 349 significantly different.

350 Fig. 4: Mean differences in WHO-5 scores according Education level and Group

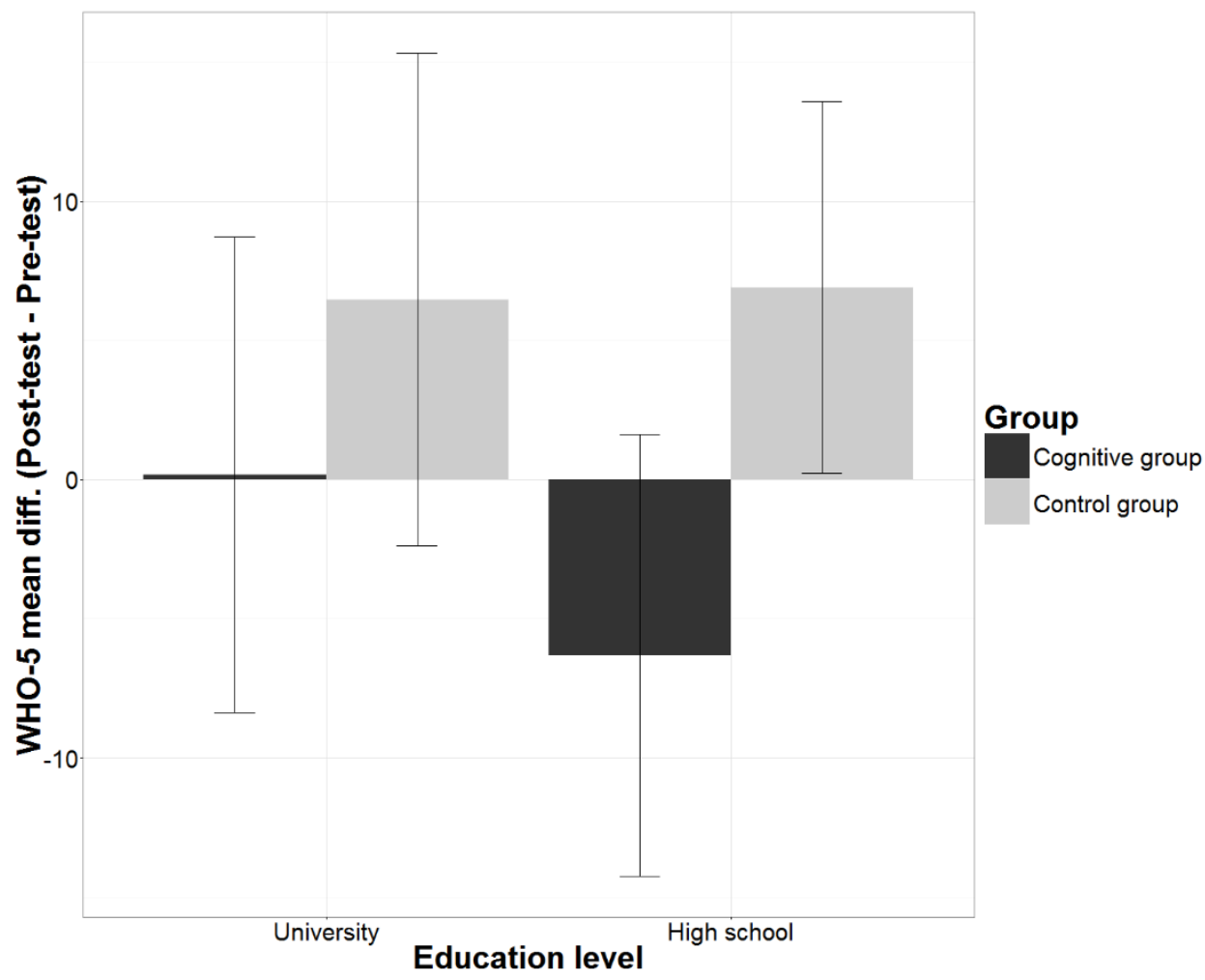

Legend: The figure shows within subject mean differences in WHO-5 scores with 95\% confidence intervals and between-subject differences split according the group and the education level.

356 There was a statistically significant interaction between Time and Group among female participants, Wilks' Lambda $=0.916, \mathrm{~F}(1,45)=4.150, \mathrm{p}=0.048$, partial eta squared $=0.084$

358 (medium effect). This indicated that women in the control group and the cognitive group responded differently to activities $(\mathrm{M}=73.95 \pm 3.93,95 \% \mathrm{CI}[66.13,81.78]$ vs $\mathrm{M}=64.18 \pm$

$3603.71,95 \% \mathrm{CI}[56.79,71.57])$. The interaction between Time and Group among male participants

361 was not significant. The marginal means plot in Fig. 5 showed that women in the cognitive group

362 declared higher baseline WHO-5 scores but lowered their well-being over the 8 week

363 intervention while women in the control group declared an increase in WHO-5 scores (Fig. 5A). 
364 A similar pattern could be observed among the male participants apart from the reversed baseline 365 scores (Fig. 5B).

366 Fig. 5: Estimated marginal means for Time*Gender*Group

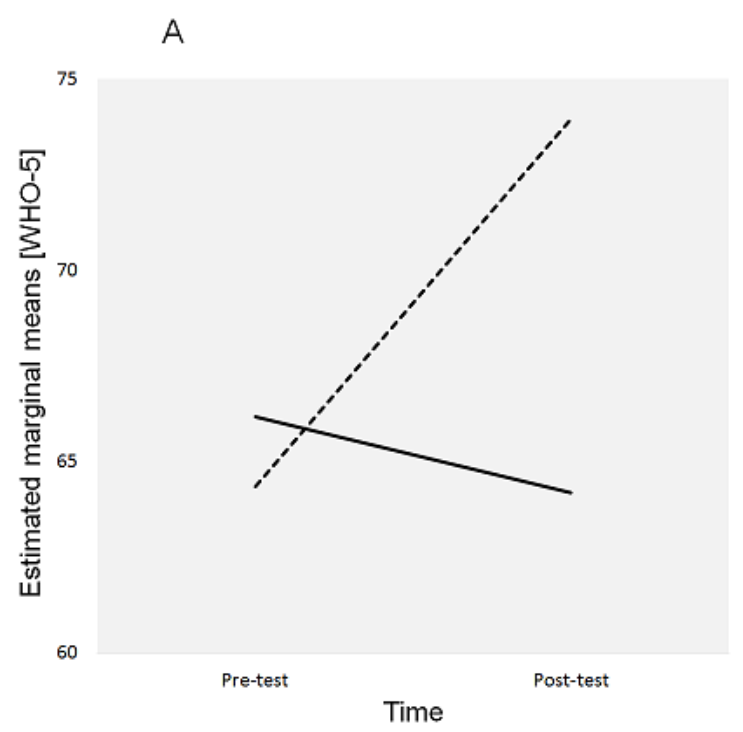

367

368

369

370

371

372

373

374

375

376

377

378

379

380 381 Gender $=$ Male. threshold.

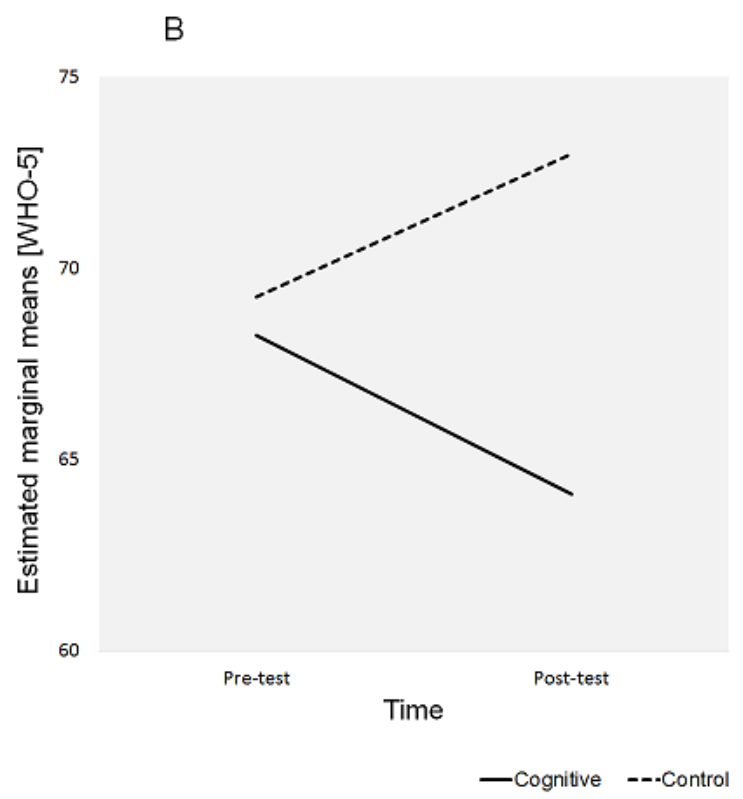

Legend: The plots show the estimated marginal means (WHO-5) for Time*Gender*Group. A) Gender $=$ Female. B)

The mean differences in WHO-5 scores in the course of intervention (Post-test - Pre-test) among female participants in the control group were $\mathrm{M}=9.61 \pm 3.79,95 \% \mathrm{CI}[2.06,17.16]$ and in the cognitive group were $\mathrm{M}=-1.98 \pm 3.58,95 \% \mathrm{CI}[-9.11,5.14]$. The mean differences in WHO-5 scores among male participants in the control group were $\mathrm{M}=3.75 \pm 4.07,95 \% \mathrm{CI}$ [$4.35,11.85]$ and in the cognitive group were $\mathrm{M}=-4.17 \pm 4.63,95 \% \mathrm{CI}[-13.39,5.06]$ (Fig. 6). The increase in WHO-5 scores among female participants in the control group was statistically significant, Wilks' Lambda $=0.919, \mathrm{~F}(1,73)=6.431, \mathrm{p}=0.013$, partial eta squared $=0.081$ (medium effect). The pairwise comparison of the means using LSD procedure revealed that resulting WHO-5 scores at the end of the intervention (Post-test) was not statistically higher compared to females in the cognitive group, $\mathrm{M}=9.77 \pm 5.40,95 \% \mathrm{CI}[-0.99,20.50], \mathrm{p}=0.059$, but the minimum mean difference of 9.77 was nearly at the $10 \%$ practical significance 


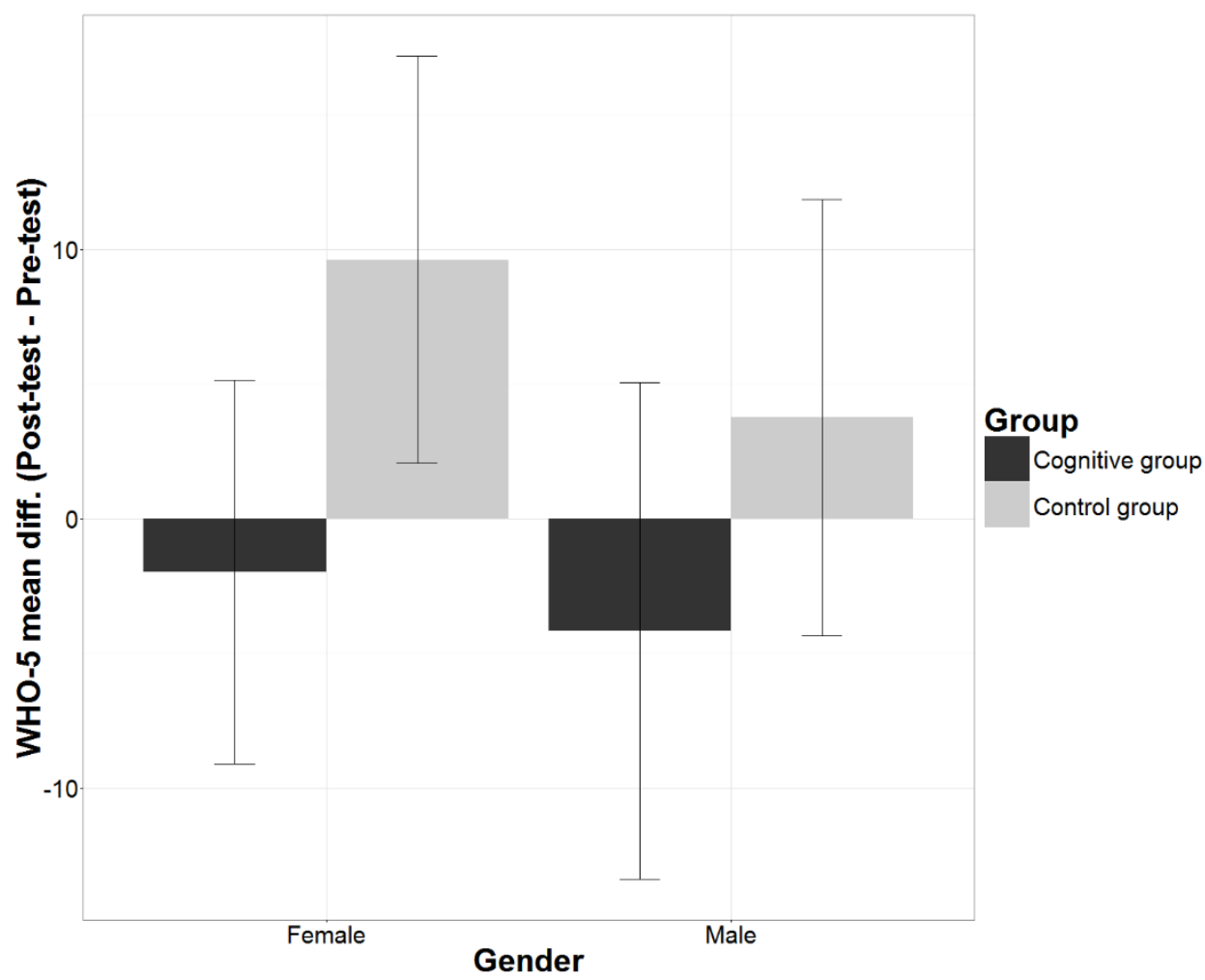

Legend: The figure shows within subject mean differences in WHO-5 scores with $95 \%$ confidence intervals and between-subject differences split according Group and Gender.

\section{Discussion}

388 This research examined the link between individualised cognitive training and the self-rated perception of well-being. The effect of individualised cognitive training was compared with leisure time activities and the moderating effects of gender and education were considered. The first major finding concerns the differences between the interventions when the effects of gender and education were statistically controlled for. The results indicated that individualised cognitive

393 training was not directly associated with improvements in well-being. Participants in the

394 cognitive group rated their well-being lower after the training intervention, but the decrease in

395 WHO-5 scores was not statistically significant. This finding is in contradiction with the assumed

396 positive effect of cognitive training on well-being and might be contrasted with the effect on

397 cognitive functions where the significant improvements in working memory and executive

398 functions were observed in the cognitive group as previously published in a study based on the 
399 same dataset (Shatil et al. 2014). The control group, who were only passing time with leisure

400 activities as distinct from strictly individualised cognitive training, showed a statistically

401 significant improvement in self-rated well-being. Although, the improvement was practically not

402 significant, falling below $10 \%$ change, it pointed out that involvement in leisure time activities

403 which participants were partly free to choose represents more favourable stimulation to a self-

404 perceived sense of well-being than individualised cognitive training despite improvements in

405 cognitive functions. Apparently, in the perspective of emotional experience, the training routine

406 and the associated stress to perform mentally challenging tasks might reduce the sense of well-

407 being.

408 The improvement in well-being might be also affected by social factors; participating in a study

409 could be a diversion from daily routine and gave a special meaning to some participants' lives.

410 This corresponds with the concept of the sense of coherence (Antonovsky 1987; Saevareid et al.

411 2007), which suggests that people involved in meaningful activities with social appraisal feel

412 better about life. The social factors, however, could not explain the differences in self-perceived

413 sense of well-being found in this study as participants in both groups received the same

414 information and the same social support.

415 The second major finding related to the moderating role of gender and education in the effect of 416 cognitive training and leisure activities on well-being. The results indicated that both gender and 417 education moderated the effect of cognitive training on well-being as compared to the effect of 418 leisure time activities. Females, regardless of their education, reacted to cognitive training and 419 leisure time activities differently. Women engaged in leisure time activities declared significant 420 increase in well-being and after the treatment their level of well-being were higher nearly at the 421 practical significance threshold compared to women in cognitive training. The reaction of males 422 follows the same pattern but did not demonstrate such significance. So, females respond with 423 more life satisfaction to diversion as reported by (Senik 2015) but when a specific performance 424 is expected their reaction is reversed.

425 In terms of education, high school graduates, regardless of gender, declare higher well-being 426 then high school graduates after cognitive training in response to leisure time activities. The 427 difference in well-being among high school participants resulted in practically significant 428 changes. University graduates did not respond to such an extent. The decreased perception of 
429 well-being among high school graduates in the cognitive group could be explained by lower 430 educated participants feeling less in control as pointed out in other studies (Ross \& Mirowsky, 431 1989; Schulz et al., 1995) and being uncomfortable under pressure inflicted by an individualised 432 training program. This fact also suggests that education can constitute an effective buffer against 433 the subjective perception of reduced well-being in some situations. These findings were 434 consistent with Zhang et al. (2011) who found that that better educated people tend to think 435 logically, rationally, and consistently, see many sides of an issue and thus are not negatively 436 impacted to the same extent by various life events as are less educated people. Similarly, 437 education implies higher income, therefore, access to better healthcare, reputable social status 438 and correspondingly positive social interactions which might also play a role in life fulfilment 439 and satisfaction (Yakovlev \& Leguizamon, 2012).

440 Since gender and education proved to moderate the effect of cognitive training, it might be 441 interesting to study the combined effect of gender and education. In this study, however, the number of participants was not high enough to achieve appropriate statistical significance. The analysis of the combined effect of gender and education is therefore referred to further research.

444 The results may have broad implications for the design of technology based training programmes 445 for elderly people. The aging population in many of the European countries directs attention to 446 cognitive training and a considerable effort has been devoted to developing technology based 447 health strategies to exploit new technological advancements (European Commission, 2012; Scott 448 \& Mars, 2013). Cognitive training delivered via the latest technology has proved to be vital in 449 reversing the adverse effect of cognitive decline (Kueider et al., 2012; Maseda et al., 2013; 450 Rizkalla, 2015; Shatil et al., 2014; van Muijden et al., 2012). However, as pointed out in this 451 study, the improved sense of self-perceived well-being is rather associated with involvement in 452 leisure activities than with improved cognitive function. Especially, being a woman, or having 453 only high school education, represents a risk in which cognitive training inflicts a decreased self454 perceived sense of well-being instead of maintaining or elevating it. Not addressing the 455 emotional dimension might reduce patients' engagement in the intervention and could be a 456 reason behind the low adherence to training regimens. Low emotional engagement limits the 457 benefits of technology assisted interventions as pointed in (Barello et al., 2015; Graffigna et al., 458 2013). It is difficult then to establish cognitive training as part of everyday life in an elderly 
459 population. Reflecting on the emotional experience during cognitive training might help to 460 improve adherence (Barello et al., 2015; Graffigna et al., 2013). These results have to be verified 461 in a follow up study in order to confirm the effect of cognitive training on the self-perceived

462 sense of well-being over an extended time-frame.

463 The present research has limitations. The findings were limited due to the fact that the control 464 group participants were involved in leisure time activities. The reason was to avoid a placebo 465 like effect among participants in the cognitive group and motivation distortion among 466 participants in the control group. Therefore, although the programmes in the cognitive and 467 control group were strictly different, from the point of view of the participants the treatment 468 seemed to be to same. Also, the recruitment procedure via advertisements in local newspapers 469 did not give an equal opportunity to all potential participants to take part in the study and the 470 study is limited to participants that responded to the advertisement, thus, the recruitment 471 procedure is prone to selection bias. Participants were also financially motivated to complete the 472 intervention which might have distorted their attitude to the administered activities. The financial 473 reward was $€ 100$ approx. (less than $€ 5$ per training session) and should have covered travel costs 474 and other out-of-pocket expenses. The results might also be influenced by activities outside the 475 protocol. These activities were recorded in participants' diaries but were not included in the 476 statistical analysis. Correspondingly, this study did not examine the influence of other potentially 477 important factors that might account for the statistically significant changes. The environment in 478 which cognitive training was conducted or the examination of specific age groups are some 479 examples. Moreover, the study also focused on healthy individuals capable of living on their 480 own. The results might differ if elderly people with mental disorders or depression were 481 considered.

\section{Conclusions}

483 In conclusion, although there are several studies proving the effect of cognitive training on 484 cognitive function, little attention has been paid to the effect of cognitive training on selfperceived well-being. This study administered individualised cognitive training and investigated the effect on emotional experience as measured by the WHO-5 index. In comparing the 
489 showed that cognitive training was not directly associated with improvements in well-being.

490 Gender and educational attainment appeared to play a role in the effect of cognitive training on

491 well-being as females and participants with only high school education declared a decreased self-

492 perceived sense of well-being after the intervention. The study opens new space for research in

493 which cognitive function and well-being are treated as different phenomena. A different

494 approach to stimulating cognitive function and a sense of well-being might help to improve

495 adherence to the cognitive training regimen. There were several limitations that should be

496 addressed in the follow up study and in future research.

497 Acknowledgments

498 The authors would like to thank all Vital Mind project members from the Philips Consumer

499 Products Innovative Lab, Czech Technical University in Prague, University of Genoa, Czech

500 TV, University of Dundee, University of Hradec Králové, Goldsmith College in London, and

501 CogniFit, the coordinator, for their valuable contribution to the project. The long term

502 development plans of UHK and FNHK are acknowledged.

503

504 References

505 Adamski N, Adler M, Opwis K, Penner IK. 2016. A pilot study on the benefit of cognitive

506 rehabilitation in Parkinson's disease. Therapeutic Advances in Neurological Disorders 9:153-

507 164. $10.1177 / 1756285616628765$

508 Allerhand M, Gale CR, Deary IJ. 2014. The dynamic relationship between cognitive function

509 and positive well-being in older people: A prospective study using the English longitudinal study

510 of aging. Psychology of Aging 29:306-318. 10.1037/a0036551

511 Antonovsky A. 1987. Unraveling the mystery of health. How people manage stress and stay

512 well. San Francisco: Jossey-Bass.

513 Ball K, Ross LA, Roth DL, Edwards JD. 2013. Speed of processing training in the ACTIVE

514 study: Who benefits? Journal of Aging and Health 25:65S-84S. 10.1177/0898264312470167 
515 Barello S, Triberti S, Graffigna G, Libreri C, Serino S, Hibbard J, Riva G. 2015. eHealth for

516 patient engagement: A systematic review. Frontiers in Psychology 6:2013.

517 10.3389/fpsyg.2015.02013

518 Bech P. 2012. Clinical Psychometrics: Oxford, UK, John Wiley \& Sons.

519 Bech P, Rasmussen NA, Olsen LR, Noerholm V, Abildgaard W. 2001. The sensitivity and 520 specificity of the Major Depression Inventory, using the Present State Examination as the index 521 of diagnostic validity. Journal of Affective Disorders 66:159-164.

522 http://dx.doi.org/10.1016/S0165-0327(00)00309-8

523 Belleville S, Gilbert B, Fontaine F, Gagnon L, Menard E, Gauthier S. 2006. Improvement of 524 episodic memory in persons with mild cognitive impairment and healthy older adults: evidence 525 from a cognitive intervention program. Dementia and Geriatric Cognitive Disorders 22:486526 499. 10.1159/000096316

527 Ben-Soussan TD, Berkovich-Ohana A, Piervincenzi C, Glicksohn J, Carducci F. 2015. 528 Embodied cognitive flexibility and neuroplasticity following Quadrato Motor Training. Frontiers 529 in Psychology 6:1021. 10.3389/fpsyg.2015.01021

530 Bier N, Grenier S, Brodeur C, Gauthier S, Gilbert B, Hudon C, Lepage E, Ouellet MC, 531 Viscogliosi C, Belleville S. 2015. Measuring the impact of cognitive and psychosocial 532 interventions in persons with mild cognitive impairment with a randomized single-blind 533 controlled trial: rationale and design of the MEMO+ study. International Psychogeriatrics 534 27:511-525. 10.1017/s 1041610214001902

535 Boot WR, Blakely DP, Simons DJ. 2011. Do action video games improve perception and 536 cognition? Frontiers in Psychology 2:226. 10.3389/fpsyg.2011.00226

537 Boquete L, Rodriguez-Ascariz JM, Amo-Usanos C, Martinez-Arribas A, Amo-Usanos J, Oton S. 538 2011. User-friendly cognitive training for the elderly: a technical report. Telemedicine Journal 539 and E-Health 17:456-460. 10.1089/tmj.2010.0149

540 Brazdil M, Ruta V, Sobotka M. 1995. Česká verze Mini-Mental State testu. Ceska Slovenska 541 Neurologie 58:244-245. 
542 Brown J, Bowling A, Flynn T. 2004. Models of quality of life: A taxonomy, overview and 543 systematic review of the literature. European Forum on Population Ageing Research. p 113 p.

544 Buiza C, Gonzalez M, Facal D, Martinez V, Diaz U, Etxaniz A, Urdaneta E, Yanguas J. 2009.

545 Efficacy of cognitive training experiences in the elderly: Can technology help? In: Stephanidis C,

546 ed. Universal Access in Human-Computer Interaction Addressing Diversity: Springer Berlin

547 Heidelberg, 324-333.

548 Calzà L, Beltrami D, Gagliardi G, Ghidoni E, Marcello N, Rossini-Favretti R, Tamburini F.

549 2015. Should we screen for cognitive decline and dementia? Maturitas 82:28-35.

550 http://dx.doi.org/10.1016/j.maturitas.2015.05.013

551 Clark DO, Xu H, Unverzagt FW, Hendrie H. 2015. Does targeted cognitive training reduce

552 educational disparities in cognitive function among cognitively normal older adults?

553 International Journal of Geriatric Psychiatry. 10.1002/gps.4395

554 Cohen-Mansfield J, Cohen R, Buettner L, Eyal N, Jakobovits H, Rebok G, Rotenberg-

555 Shpigelman S, Sternberg S. 2015. Interventions for older persons reporting memory difficulties:

556 a randomized controlled pilot study. International Journal of Geriatric Psychiatry 30:478-486.

$557 \quad 10.1002 /$ gps.4164

558 Cohen J, Cohen P, West SG, Aiken LS. 2002. Applied multiple regression/Correlation analysis 559 for the behavioral sciences. New Jersey: Routledge.

560 Crum RM, Anthony JC, Bassett SS, Folstein MF. 1993. Population-based norms for the Mini-

561 Mental State Examination by age and educational level. JAMA 269:2386-2391.

562 Eiser C, Morse R. 2001. Quality-of-life measures in chronic diseases of childhood. Health

563 Technology Assessment, 5.

564 Elgamal SA, Roy EA, Sharratt MT. 2011. Age and verbal fluency: the mediating effect of speed 565 of processing. Canadian Geriatrics Journal 14:66-72. 10.5770/cgj.v14i3.17

566 European Commission. 2012. eHealth Action Plan 2012-2020 - Innovative healthcare for the 56721 st century. Communication from the Commission to the European Parliament, the Council, the 
568 European Economic and Social Committee and the Committee of the Regions. Brussels, $569 \quad 6.12 .2012$.

570 Folstein MF, Folstein SE, McHugh PR. 1975. "Mini-mental state". A practical method for

571 grading the cognitive state of patients for the clinician. Journal of Psychiatric Research 12:189572198.

573 Gaiswinkler L, Unterrainer HF. 2016. The relationship between yoga involvement, mindfulness 574 and psychological well-being. Complementary Therapies in Medicine 26:123-127.

575 10.1016/j.ctim.2016.03.011

576 Gates NJ, Valenzuela M, Sachdev PS, Singh NA, Baune BT, Brodaty H, Suo C, Jain N, Wilson 577 GC, Wang Y, Baker MK, Williamson D, Foroughi N, Fiatarone Singh MA. 2011. Study of 578 Mental Activity and Regular Training (SMART) in at risk individuals: A randomised double 579 blind, sham controlled, longitudinal trial. BMC Geriatrics 11:19-19. 10.1186/1471-2318-11-19

580 Getzmann S, Golob EJ, Wascher E. 2016. Focused and divided attention in a simulated cocktail581 party situation: ERP evidence from younger and older adults. Neurobiology of Aging 41:138582 149. 10.1016/j.neurobiolaging.2016.02.018

583 Gigler KL, Blomeke K, Shatil E, Weintraub S, Reber PJ. 2013. Preliminary evidence for the 584 feasibility of at-home online cognitive training with older adults. Gerontechnology 12:26-35. $58510.4017 / g t .2013 .12 .1 .007 .00$

586 Goyal M, Singh S, Sibinga EM, Gould NF, Rowland-Seymour A, Sharma R, Berger Z, Sleicher 587 D, Maron DD, Shihab HM, Ranasinghe PD, Linn S, Saha S, Bass EB, and Haythornthwaite JA. 588 2014. Meditation programs for psychological stress and well-being: a systematic review and 589 meta-analysis. JAMA Internal Medicine 174:357-368. 10.1001/jamainternmed.2013.13018

590 Graffigna G, Barello S, Riva G. 2013. How to make health information technology effective: the 591 challenge of patient engagement. Archives Physical Medicine and Rehabilitation 94:2034-2035. 592 10.1016/j.apmr.2013.04.024

593 Graham C, Chattopadhyay S. 2013. Gender and wellbeing around the world. International 594 Journal of Happiness and Development 1:212-232. 
595 Henry JD, Cowan DG, Lee T, Sachdev P. 2015. A review of different methods of assessing 596 social cognitive function in clinical settings. Current Opinion Psychiatry.

$597 \quad 10.1097 /$ yco.0000000000000139

598 Huppert FA, Whittington JE. 2003. Evidence for the independence of positive and negative well599 being: implications for quality of life assessment. British Journal of Health Psychology 8:107$600 \quad 122.10 .1348 / 135910703762879246$

601 Innes KE, Selfe TK, Khalsa DS, Kandati S. 2016. A randomized controlled trial of two simple 602 mind-body programs, Kirtan Kriya meditation and music listening, for adults with subjective 603 cognitive decline: Feasibility and acceptability. Complementary Therapies in Medicine 26:98604 107. http://dx.doi.org/10.1016/j.ctim.2016.03.002

605 Isaacowitz DM, Smith J. 2003. Positive and negative affect in very old age. The Journals of 606 Gerontology Series B, Psychological Sciences and Social Sciences 58:P143-152.

607 Jansen P, Dahmen-Zimmer K. 2012. Effects of cognitive, motor, and karate training on cognitive 608 functioning and emotional well-being of elderly People. Frontiers in Psychology 3:40.

609 10.3389/fpsyg.2012.00040

610 Jost K, Bryck RL, Vogel EK, Mayr U. 2011. Are old adults just like low working memory young 611 adults? Filtering efficiency and age differences in visual working memory. Cerebral Cortex 612 21:1147-1154. 10.1093/cercor/bhq185

613 Ko PC, Duda B, Hussey E, Mason E, Molitor RJ, Woodman GF, Ally BA. 2014. Understanding 614 age-related reductions in visual working memory capacity: examining the stages of change 615 detection. Attention, Perception, \& Psychophysics 76:2015-2030. 10.3758/s13414-013-0585-z

616 Kueider AM, Parisi JM, Gross AL, Rebok GW. 2012. Computerized cognitive training with 617 older adults: a systematic review. PLoS One 7:e40588. 10.1371/journal.pone.0040588

618 Llewellyn DJ, Lang IA, Langa KM, Huppert FA. 2008. Cognitive function and psychological 619 well-being: findings from a population-based cohort. Age and Ageing 37:685-689.

620 10.1093/ageing/afn194 
621 Lun VMC, Bond MH. 2016. Achieving subjective well-being around the world: The moderating 622 influence of gender, age and national goals for socializing children. Journal of Happiness Studies 623 17:587-608. 10.1007/s10902-015-9614-Z

624 Manenti R, Sandrini M, Brambilla M, Cotelli M. 2016. The optimal timing of stimulation to 625 induce long-lasting positive effects on episodic memory in physiological aging. Behavioural 626 Brain Research 311:81-86. 10.1016/j.bbr.2016.05.028

627 Maseda A, Millan-Calenti JC, Lorenzo-Lopez L, Nunez-Naveira L. 2013. Efficacy of a 628 computerized cognitive training application for older adults with and without memory 629 impairments. Aging Clinical and Experimental Research 25:411-419. 10.1007/s40520-013$630 \quad 0070-5$

631 McDowell I. 2010. Measures of self-perceived well-being. Journal of Psychosomatic Research 632 69:69-79. http://dx.doi.org/10.1016/j.jpsychores.2009.07.002

633 Missotten P, Squelard G, Ylieff M, Di Notte D, Paquay L, De Lepeleire J, Buntinx F, Fontaine 634 O. 2008. Relationship between quality of life and cognitive decline in dementia. Dementia and 635 Geriatric Cognitive Disorders 25:564-572. 10.1159/000137689

636 Nana GN, Doulougou B, Gomez F, Ylli A, Guralnik J, Zunzunegui MV. 2015. Social differences 637 associated with the use of psychotropic drugs among men and women aged 65 to 74 years living 638 in the community: the International Mobility in Aging Study (IMIAS). BMC Geriatrics 15:85. $63910.1186 / \mathrm{s} 12877-015-0083-3$

640 O'Bryant SE, Humphreys JD, Smith GE, Ivnik RJ, Graff-Radford NR, Petersen RC, Lucas JA. 641 2008. Detecting Dementia with the Mini-Mental State Examination (MMSE) in Highly Educated 642 Individuals. Archives of Neurology 65:963-967. 10.1001/archneur.65.7.963

643 Olsen LR, Jensen DV, Noerholm V, Martiny K, Bech P. 2003. The internal and external validity 644 of the Major Depression Inventory in measuring severity of depressive states. Psychological 645 Medicine 33:351-356. 
646 Peretz C, Korczyn AD, Shatil E, Aharonson V, Birnboim S, Giladi N. 2011. Computer-based, 647 personalized cognitive training versus classical computer games: a randomized double-blind 648 prospective trial of cognitive stimulation. Neuroepidemiology 36:91-99. 10.1159/000323950

649 Pusswald G, Tropper E, Kryspin-Exner I, Moser D, Klug S, Auff E, Dal-Bianco P, Lehrner J. 650 2015. Health-related quality of life in patients with subjective cognitive decline and mild 651 cognitive impairment and its relation to activities of daily living. Journal of Alzheimer's Disease 652 47:479-486. 10.3233/jad-150284

653 Rapacciuolo A, Perrone Filardi P, Cuomo R, Mauriello V, Quarto M, Kisslinger A, Savarese G, 654 Illario M, Tramontano D. 2016. The impact of social and cultural engagement and dieting on 655 well-being and resilience in a group of residents in the metropolitan area of Naples. Journal of 656 Aging Research 2016:4768420. 10.1155/2016/4768420

657 Ready RE, Ott BR, Grace J, Cahn-Weiner DA. 2003. Apathy and executive dysfunction in mild 658 cognitive impairment and Alzheimer disease. The American Journal of Geriatric Psychiatry $659 \quad 11: 222-228$.

660 Rizkalla MN. 2015. Cognitive training in the elderly: a randomized trial to evaluate the efficacy 661 of a self-administered cognitive training program. Aging \& Mental Health1-11.

$66210.1080 / 13607863.2015 .1118679$

663 Ross CE, Mirowsky J. 1989. Explaining the social patterns of depression: control and problem 664 solving--or support and talking? Journal of Health and Social Behavior 30:206-219.

665 Ryan RM, Deci EL. 2001. On happiness and human potentials: a review of research on hedonic 666 and eudaimonic well-being. Annual Review of Psychology 52:141-166.

667 10.1146/annurev.psych.52.1.141

668 Saevareid HI, Thygesen E, Nygaard HA, Lindstrom TC. 2007. Does sense of coherence affect 669 the relationship between self-rated health and health status in a sample of community-dwelling 670 frail elderly people? Aging \& Mental Health 11:658-667. 10.1080/13607860701368513 
671 Schulz AJ, Israel BA, Zimmerman MA, Checkoway BN. 1995. Empowerment as a multi-level 672 construct: perceived control at the individual, organizational and community levels. Health 673 Education Research 10:309-327. 10.1093/her/10.3.309

674 Scott RE, Mars M. 2013. Principles and framework for eHealth strategy development. Journal of 675 Medical Internet Research 15:e155. 10.2196/jmir.2250

676 Senik C. 2015. Gender gaps in subjective wellbeing. European Commission - Directorate677 General for Justice.

678 Shatil E. 2013. Does combined cognitive training and physical activity training enhance 679 cognitive abilities more than either alone? A four-condition randomized controlled trial among 680 healthy older adults. Frontiers in Aging Neuroscience 5:8. 10.3389/fnagi.2013.00008

681 Shatil E, Mikulecká J, Bellotti F, Bureš V. 2014. Novel Television-Based Cognitive Training 682 Improves Working Memory and Executive Function. PLoS One 9:e101472.

683 10.1371/journal.pone.0101472

684 Simoni-Wastila L. 2000. The use of abusable prescription drugs: the role of gender. Journal of 685 Women's Health \& Gender Based Medicine 9:289-297. 10.1089/152460900318470

686 Skead NK, Rogers SL. 2016. Running to well-being: A comparative study on the impact of 687 exercise on the physical and mental health of law and psychology students. International Journal 688 of Law and Psychiatry. 10.1016/j.ijlp.2016.05.012

689 Smith GE. 2016. Healthy cognitive aging and dementia prevention. The American Psychologist $69071: 268-275.10 .1037 / \mathrm{a} 0040250$

691 Sperduti M, Makowski D, Piolino P. 2016. The protective role of long-term meditation on the 692 decline of the executive component of attention in aging: a preliminary cross-sectional study. 693 Neuropsychology, Development and Cognition Section B, Aging Neuropsychology and Cognition 694 1-12. 10.1080/13825585.2016.1159652

695 Stevenson B, Wolfers J. 2009. The paradox of declining female happiness. National Bureau of 696 Economic Research Working Paper Series No. 14969. 10.3386/w14969 
697 Stogmann E, Moser D, Klug S, Gleiss A, Auff E, Dal-Bianco P, Pusswald G, Lehrner J. 2015.

698 Activities of daily living and depressive symptoms in patients with subjective cognitive decline,

699 Mild cognitive impairment, and Alzheimer's disease. Journal of Alzheimer's Disease 49:1043-

700 1050. 10.3233/jad-150785

701 Taylor SE, Brown JD. 1988. Illusion and well-being: A social psychological perspective on 702 mental health. Psychological Bulletin 103:193-210.

703 Tian Q. 2016. Intergeneration social support affects the subjective well-being of the elderly:

704 Mediator roles of self-esteem and loneliness. Journal of Health Psychology 21:1137-1144.

$705 \quad 10.1177 / 1359105314547245$

706 Topp CW, Østergaard SD, Søndergaard S, Bech P. 2015. The WHO-5 Well-Being Index: A

707 systematic review of the Literature. Psychotherapy and Psychosomatics 84:167-176.

708 Tromp D, Dufour A, Lithfous S, Pebayle T, Després O. 2015. Episodic memory in normal aging 709 and Alzheimer disease: Insights from imaging and behavioral studies. Ageing Research Reviews

710 24, Part B:232-262. http://dx.doi.org/10.1016/j.arr.2015.08.006

711 Turgeon M, Lustig C, Meck WH. 2016. Cognitive aging and time perception: Roles of Bayesian

712 optimization and degeneracy. Frontiers in Aging Neuroscience 8:102. 10.3389/fnagi.2016.00102

713 van Muijden J, Band GP, Hommel B. 2012. Online games training aging brains: limited transfer

714 to cognitive control functions. Frontiers in Human Neuroscience 6:221.

$715 \quad 10.3389 /$ fnhum.2012.00221

716 Walker A, Lowenstein A. 2009. European perspectives on quality of life in old age. European

717 Journal of Ageing 6:61-66. 10.1007/s10433-009-0117-9

718 WHO. 1998. WHO-5 Well-being Index (Czech version). Available at http://www.who-5.org/.

719 WHO. 2011. Mental health: a state of well-being. World Health Organization.

720 WHO. 2015. Gender and women's mental health. Available at

721 http://www.who.int/mental_health/prevention/genderwomen/en/ (accessed 29 August, 2015. 
722 Willis SL, and Caskie GIL. 2013. Reasoning training in the ACTIVE study: How much is 723 needed and who benefits? Journal of Aging and Health 25:10.1177/0898264313503987.

$724 \quad 10.1177 / 0898264313503987$

725 Yakovlev P, Leguizamon S. 2012. Ignorance is not bliss: On the role of education in subjective 726 well-being. The Journal of Socio-Economics 41:806-815.

727 http://dx.doi.org/10.1016/j.socec.2012.08.009

728 Zhang W, Chen Q, McCubbin H, McCubbin L, Foley S. 2011. Predictors of mental and physical 729 health: Individual and neighborhood levels of education, social well-being, and ethnicity. Health 730 \& Place 17:238-247. http://dx.doi.org/10.1016/j.healthplace.2010.10.008 